\title{
Novel regional age-associated DNA methylation changes within human common disease-associated loci
}

\author{
Christopher G. Bell ${ }^{1,2,3,4^{*}}$, Yudong Xia ${ }^{5}$, Wei Yuan ${ }^{1,6}$, Fei Gao ${ }^{5}$, Kirsten Ward ${ }^{1}$, Leonie Roos ${ }^{1}$, Massimo Mangino ${ }^{1}$, \\ Pirro G. Hysi ${ }^{1}$, Jordana Bell ${ }^{1}$, Jun Wang ${ }^{5}$ and Timothy D. Spector ${ }^{1}$
}

\begin{abstract}
Background: Advancing age progressively impacts on risk and severity of chronic disease. It also modifies the epigenome, with changes in DNA methylation, due to both random drift and variation within specific functional loci.

Results: In a discovery set of 2238 peripheral-blood genome-wide DNA methylomes aged 19-82 years, we identify 71 age-associated differentially methylated regions within the linkage disequilibrium blocks of the single nucleotide polymorphisms from the $\mathrm{NIH}$ genome-wide association study catalogue. This included 52 novel regions, 29 within loci not covered by 450 k or 27 k Illumina array, and with enrichment for DNase-I Hypersensitivity sites across the full range of tissues. These age-associated differentially methylated regions also show marked enrichment for enhancers and poised promoters across multiple cell types. In a replication set of 2084 DNA methylomes, $95.7 \%$ of the age-associated differentially methylated regions showed the same direction of ageing effect, with $80.3 \%$ and $53.5 \%$ replicated to $p<0.05$ and $p<1.85 \times 10^{-8}$, respectively.

Conclusion: By analysing the functionally enriched disease and trait-associated regions of the human genome, we identify novel epigenetic ageing changes, which could be useful biomarkers or provide mechanistic insights into age-related common diseases.
\end{abstract}

Keywords: Epigenetics, DNA methylation, Ageing, Common Disease, Human

\section{Background}

Age is a risk factor for multiple chronic diseases. It impacts on all organ systems leading to decreased functionality and eventual death [1]. Epigenetic changes associated with the ageing process, specifically DNA methylation, have been identified in a number of studies [2-16]. While stochastic epigenetic drift does occur [17], non-random changes are seen within specific functional loci, such as increased DNA methylation in the promoters of the target genes of polycomb group proteins [3] and bivalent chromatin regions or poised promoters [4]. Additionally, recent formulation of an epigenetic

\footnotetext{
* Correspondence: christopher.g.bell@kcl.ac.uk; cgb@mrc.soton.ac.uk 'Department of Twin Research \& Genetic Epidemiology, King's College London, London, UK

${ }^{2}$ MRC Lifecourse Epidemiology Unit, University of Southampton,

Southampton, UK

Full list of author information is available at the end of the article
}

'clock' [9] has led to the observation that accelerated discordance between the DNA methylation estimate and actual chronological age is a risk factor for all-cause mortality in later life [18].

The ageing-associated DNA methylation signatures identified to date have almost exclusively been via targeted array based methodology (Illumina $27 \mathrm{k}$ or $450 \mathrm{k}$ ). Notable exceptions are a study by McClay et al. performed via methyl binding domain enriched second-generation sequencing (MBD-seq) [12] and Heyn et al. comparing a single centenarian with a single newborn with whole genome bisulphite sequencing (WGBS) data [6]. Within heterogeneous peripheral blood, an increase in the myeloid fraction of blood cell sub-types is known to occur with advancing age [19] and must be accounted for in these analyses. DNA methylation changes in peripheral blood may therefore represent this myeloid skewing, biological 
cascades leading to upregulation or downregulation of specialised cell subtypes [20], or additional undefined active or passive changes associated with age [21].

We analysed 2238 unique DNA methylomes for changes associated with chronological age. These data were generated by genome-wide methylated DNA immunoprecipitation Illumina second-generation sequencing (MeDIP-seq) in peripheral blood. MeDIP-seq methylome results identify broader regional changes compared with targeted individual $\mathrm{CpG}$ array approaches and are not limited to the array-predefined cytosines. Data were included on significant influencing variables including full blood count, smoking status and batch. To account for the strong genetic effects inherent with DNA methylation analysis, especially by techniques such as MeDIP-seq [22] we incorporated common SNP genotyping data available on all these individuals.

We focused on the a priori functional regions [23], the linkage disequilibrium (LD) blocks of all of the phenotype and disease-related single-nucleotide polymorphisms (SNPs) entered in the genome-wide association study (GWAS) catalogue [24] (8093 curated GWAS SNPs with $p$ value $<1 \times 10^{-7}$ residing within 2709 distinct LD blocks, $\sim 22.1 \%$ of the genome). Multiple strands of evidence have now accrued from the ENCODE consortium and other regulatory datasets that the regions identified through the thousands of GWAS studies performed to date are enriched for active loci $[25,26]$. We pursued this strategy to explore the GWAS LD blocks in order to identify novel epigenetic changes that were more likely to be functional and, due to their co-location, would enable direct integration into future locus-specific common disease investigations. As age impacts on the penetrance and severity of many of these common diseases and phenotypes, this analysis enabled us to specifically explore these blocks for agerelated changes. This is particularly of interest because changes identified in studies, such as the DNA methylation 'clock' and others, can be seen across multiple tissues [9, 15]. Thus these blood-based findings could, in some cases, have impact not just in haematological or immunological disorders, but also within the most disease-relevant tissue.

The epigenetic state within a locus can be obligatory or fixed due to the underlying genetic framework or may vary, facilitated by particular sequence constructs [27]. Using haplotype-tagging common SNP data in these individuals, we could control for the obligatory genetic effects in the LD blocks. We could also subsequently investigate whether facilitative DNA methylation ageing changes differed with respect to common risk versus non-risk haplotype background within these GWAS regions. The potential of genetically facilitated ageing modifications has been explored [10, 16] and its direct assessment could add additional mechanistic insight within these disease-associated loci.

\section{Results}

GWAS LD block regions are functionally enriched

Multiple studies have indicated that the regions identified by GWAS are functionally enriched $[25,26]$. To further demonstrate this, we explored public ENCODE data and compared the 2709 distinct GWAS LD blocks ( $22.1 \%$ of the genome) to the remainder of the genome. We focused on the DNase I Hypersensitivity site (DHS) due to their broad capacity to act as functional indicators [28]. We also explored the recently identified DNA methylation-sensitive transcription factor NRF1 [29]. We found that DHSs and NRF1 both show significant occupancy enrichment within this portion of the genome (both Fisher's exact test $p<2.2 \times 10^{-16}$, odds ratio $(\mathrm{OR})=1.70$ and 2.26 , confidence intervals $(\mathrm{CI})$ $1.69-1.71$ and $2.17-2.35$, respectively).

\section{Age-associated differentially methylated regions}

From our discovery sample set of 2238 MeDIP-seq DNA methylomes, we identified 115 individual 500-bp windows that passed a Bonferroni corrected significance level $\left(p<1.85 \times 10^{-8}\right.$, Fig. 1; bidirectional Manhattan plot) within these GWAS LD block regions. Due to overlapping and adjacent windows, these merged into 71 discrete age-associated differentially methylated regions (a-DMRs) (Additional file 1). The a-DMRs were $54.9 \%$ and $45.1 \%$ hypermethylated and hypomethylated with increasing age, respectively. They were on average the size of $\sim 0.65 \mathrm{~kb}$ and include 1546 individual CpGs.

To ascertain the novelty of our results, we compared the 71 a-DMRs with results from 14 prior studies also performed in blood [3-16]. All these studies were arraybased and therefore focused on a defined set of CpGs, except for McClay et al. (MBD-seq) [12] and Heyn et al. (WGBS) [6]. The array-based studies are less in sample size, except for a meta-analysis [9], with a maximum of around 1000 individuals, but the majority is much smaller. In total, 52 (73.2 \%) of our a-DMRs were previously unidentified associations with age (Additional file 2: Table S1). Highlighting the use of the MeDIP-seq data, 29 of the a-DMR regions identified $(40.8 \%)$ were not covered at all by any CpG probe from either the $27 \mathrm{k}$ or $450 \mathrm{k}$ array platforms. A further $11 \mathrm{a}$-DMRs are covered on these arrays by only one or two CpGs.

\section{a-DMR chromatin segmentation is enriched for poised promoters and enhancers}

To identify chromatin-defined epigenomic functional enrichments, the a-DMRs were compared with both ChromHMM [30] and the Combined (ChromHMM and Segway [31]) genome segmentation analysis from nine and six tissue types, respectively (Fig. $2 \mathrm{a}$ and b). This was in a comparison with the GWAS LD blocks (using 500-bp non-overlapping windows), not the entire 


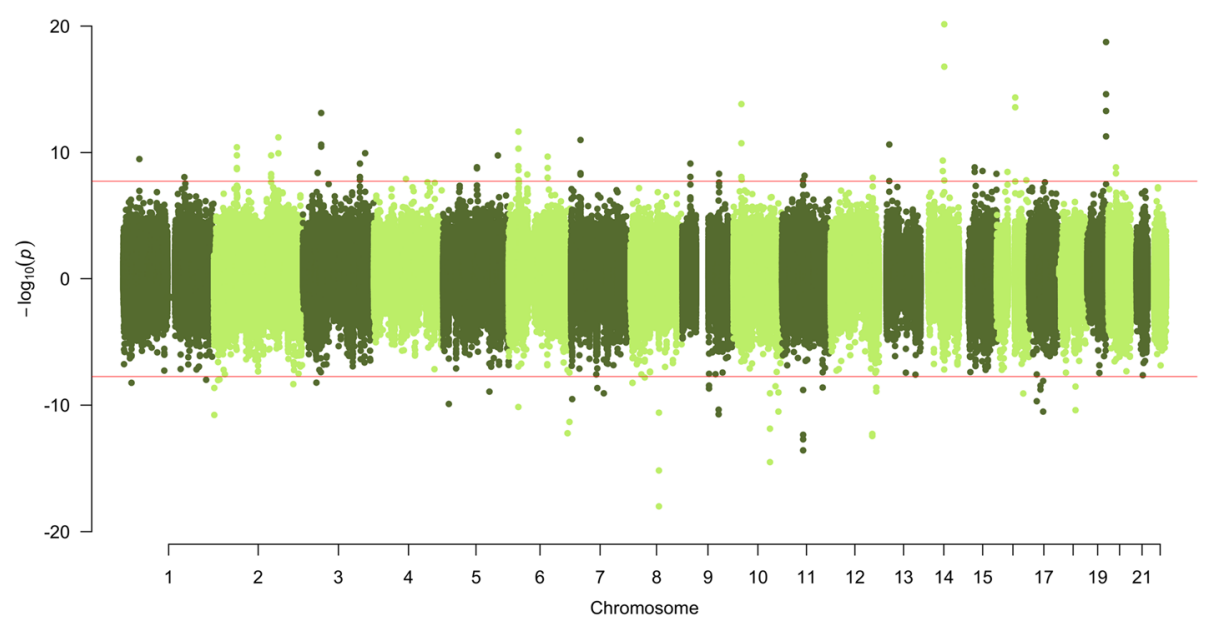

Fig. 1 Bidirectional Manhattan plot of age-associated differentially methylated regions (a-DMRs). Loci with a positive correlated with age are displayed in the positive $y$-axis and those regions where DNA methylation changes negatively correlate with age are displayed in the negative $y$-axis
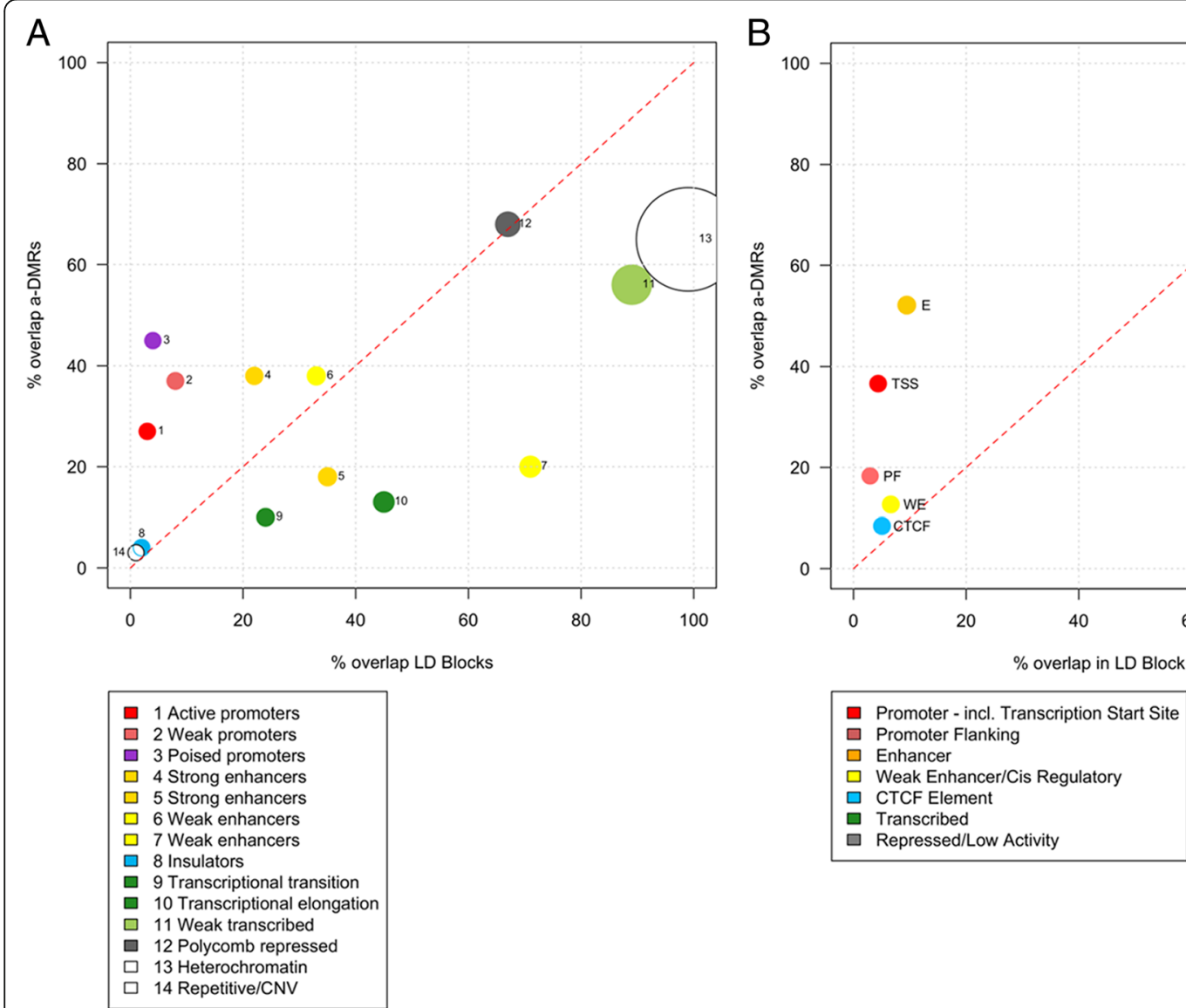

Fig. 2 Chromatin segmentation enrichment. a Overlap with a-DMRs and GWAS LD blocks for ENCODE ChromHMM [30] genome segmentation from ENCODE in nine tissues (data via and graph adapted from Epiexplorer [85]). Strong poised promoter enrichment seen. Observed versus expected for the GWAS LD block regions. b Overlap with a-DMRs and LD blocks for combined genome segmentation from ENCODE (ChromHMM [30] and Segway [31]) in six tissues. Strong enrichment for enhancers is evident. Sphere size is proportional to genomic space. TSS predicted promoter region including transcription start site, PF predicted promoter flanking region, E predicted enhancer, WE predicted weak enhancer or open chromatin cis regulatory element, CTCF CTCF enriched element, $T$ predicted transcribed region, $R$ predicted repressed or low activity region 
genome, as these regions already have an inherent functional increase [23]. Ageing changes have previously been identified in poised promoters [4] and in this ChromHMM analysis, this enrichment was particularly strong (Fig. 2a, $\sim 45 \% c f . \sim 3 \%$ within LD blocks, $\left.\chi^{2} p<2.2 \times 10^{-16}\right)$. Strong and weak promoters are also more prevalent, but interestingly, there is a separation between the delineated ChromHMM enhancer classes. Enhancer states 4 and 6 show increases, however 5 and 7 do not. The major constituent difference between these classes is higher levels of H3K4me2 (state 4: $99 \%$ and state 6: $75 \%$ versus state 5: $57 \%$ and state 7: $3 \%$ ) and recent data from Wang et al. indicate that specifically high levels of H3K4me2 reliably identify transcription factor binding regions across different cells [32].

As stated we also compared the a-DMRs with the combined segmentation that incorporates overlap with the Segway algorithm and results in more discrete, reduced and potentially more accurate categories [31] (Fig. 2b). This classification does not include the poised promoter subcategory. It classifies an extremely strong enrichment in enhancer sequence across these multiple cell types (52.1\% a-DMRs versus $9.46 \%$ in LD block non overlapping 500-bp windows, $\chi^{2} p<2.2 \times 10^{-16}$ ).

\section{a-DMRs are enriched for genetic functional indicators}

We then examined additional specified genetic and functional regions, in the same manner as the segmentation analysis above, by again comparison with the regions within the GWAS LD blocks, not the entire genome. This revealed greater fold enrichment for a-DMRs within CpG Islands (CGI) themselves (14.33-fold), even stronger, in fact, than for CpG Island shores (7.22-fold; Fig. 3). Thus a-DMRs differ from the findings in tissue-specific [33], reprogramming-specific [34] and species-specific [35] DMRs which are more prevalent in CGI shores. a-DMRs also showed enrichment within functional indicators such as DNase I hypersensitivity sites and transcription factor ChIP-seq binding sites, as well as even stronger fold enrichments for CTCF and Sp1 ENCODE ChIP-seq data across all tissues $\left(\chi^{2} p\right.$ all $\left.<1 \times 10^{-10}\right)$. The eRNA expression validated FANTOM5 enhancer set [36] also showed a strong increase for a-DMR locations $\left(\chi^{2} p=2.2 \times 10^{-16}\right)$. Repeat classes in total were significantly depleted by comparison $\left(x^{2} p=6.70 \times 10^{-9}\right)$, although potential mapping issues to these regions may confound this.

\section{a-DMR Gene Ontology analysis}

Gene Ontology enrichment was performed with the Genomic Regions Enrichment of Annotations Tool (GREAT) comparing the a-DMRs to the region contained within the LD blocks as the background set (Additional file 2: Table S2). This revealed an increase for categories (all Bonferroni $p<0.05$ ), such as Molecular

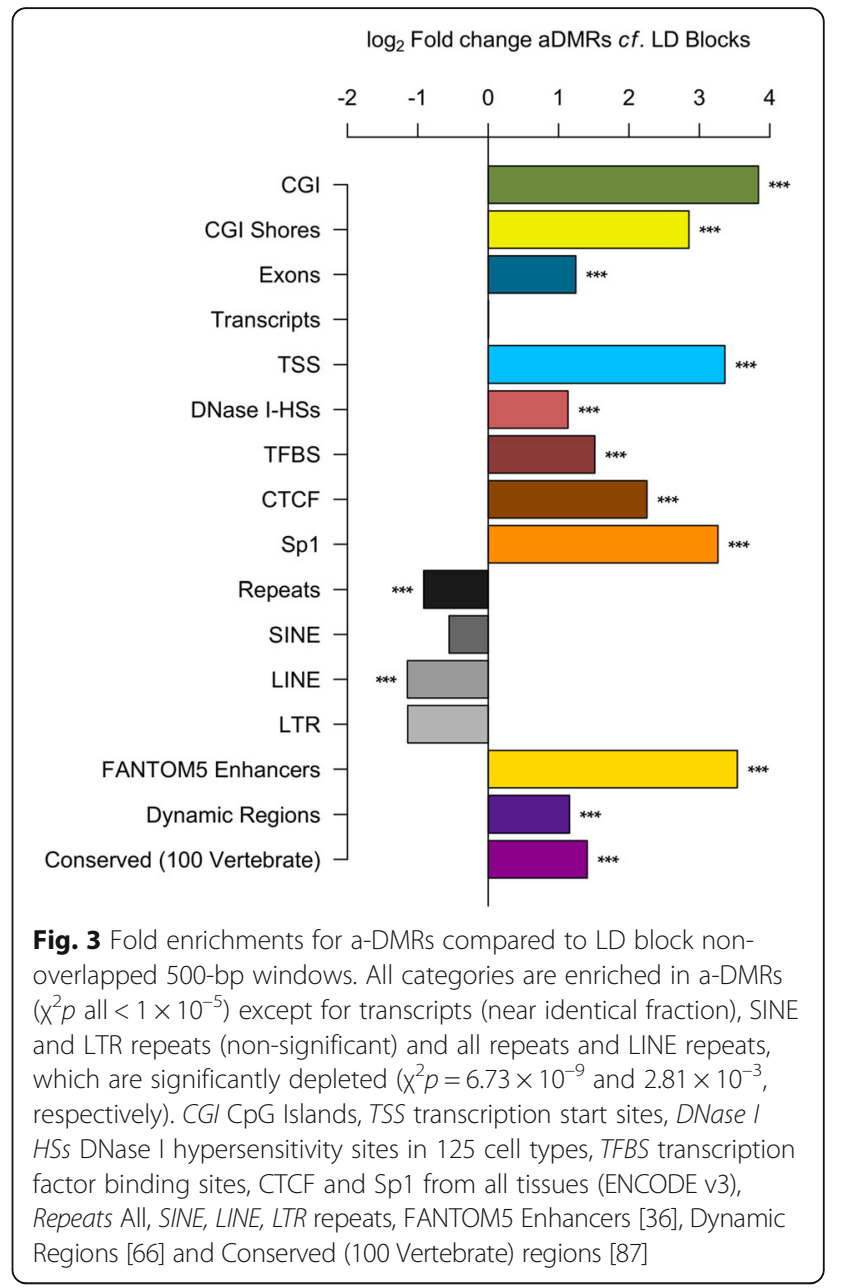

classifications of DNA binding; Nucleic acid binding transcription factor activity; Sequence-specific DNA binding transcription factor activity; and Nucleic acid binding. An enrichment in the PRD Gene family was also identified which includes multiple homeobox genes.

\section{Hypermethylated and hypomethylated a-DMR transcription factor binding site analysis}

We then examined the sequence within the 38 hypermethylated and 33 hypomethylated a-DMRs separately and explored for sequence enrichment of specific transcription factor binding sites (TFBSs) with both the transcription factor affinity prediction (TRAP) [37] and MEME-ChIP algorithms. [38] TRAP identified four TFBSs, three within hypomethylated DMRs including NFE2L2 $\quad(p<0.05, \quad$ Benjamini-Hochberg corrected; Table 1), previously associated with age-related diseases [39]. The MEME analysis, which identifies sequences agnostically, before then comparing them to known motifs in TOMTOM, found three sequences in hypomethylated a-DMRs, that were found to closely match a number of motifs and only one match for 
Table 1 TRAP transcription factor motif prediction

\begin{tabular}{llllll}
\hline Rank & $\begin{array}{l}\text { Combined } \\
p \text { value }\end{array}$ & $\begin{array}{l}\text { Corrected } p \text { value } \\
\text { (Benjamini-Hochberg) }\end{array}$ & Matrix ID & Motif & a-DMR \\
\hline 1 & $3.84 \times 10^{-6}$ & $4.53 \times 10^{-4}$ & MA0150.1 & NFE2L2 & Hypo \\
2 & $1.52 \times 10^{-5}$ & $8.97 \times 10^{-4}$ & MA0041.1 & FOXI1 & Hypo \\
3 & $4.13 \times 10^{-5}$ & $1.63 \times 10^{-3}$ & MA0041.1 & FOXD3 & Hypo \\
1 & $1.99 \times 10^{-5}$ & $2.35 \times 10^{-3}$ & MA0152.1 & NFATC2 & Hyper \\
\hline
\end{tabular}

Enrichment for defined TFBS within hypomethylated and hypermethylated a-DMRs via the TRAP algorithm [37]

hypermethylated a-DMRs (Table 2). These results included the SP1 motif, that is of interest as it corresponds with the ENCODE TF CHIP-seq enrichment data and also due to the known methylation-determining region effect of this TFBS [40] (Fig. 4). An enrichment for the binding motif of KLF14 is noteworthy due this gene's known role as a master trans-regulator in metabolism [41].

\section{No enrichment with blood-cell derived DNA methylome changes}

The myeloid skew with age is an acknowledged confounding factor in the analysis of ageing changes. However, Yuan et al. have presented data that the majority of age-related drift is independent of increase of granulocytes in comparison to lymphocytes [42].

We had included available leukocyte fraction data in our linear mixed-effect model, but to further test whether we had accounted for these effects we performed additional investigations. We first compared our a-DMRs with the 500 leukocyte subtype-related differentially methylated CpG positions (L-DMPs) identified by Houseman et al. [43]. None of the a-DMRs overlap with these L-DMPs.

Table 2 MEME/TOMTOM agnostic motif analysis

\begin{tabular}{ll}
\hline Hypomethylated motif 1 & SPDEF_DBD_3 \\
Hypomethylated motif 2 & FOXC1_DBD_1 \\
& ONECUT3_DBD \\
& UP00037_1 (Zfp105_primary) \\
& UP00097_2 (Mtf1_secondary) \\
& ONECUT1_DBD \\
& SP8_DBD \\
Hypomethylated motif 3 & SP1_DBD \\
& SP3_DBD \\
& KLF14_DBD \\
& KIf12_DBD \\
& KLF16_DBD \\
& SP4_full \\
& UP00011_2 (Irf6_secondary). \\
& SPDEF_DBD_3
\end{tabular}

Enrichment for defined TFBS within hypomethylated and hypermethylated a-DMRs via MEME/TOMTOM motif analysis [38]

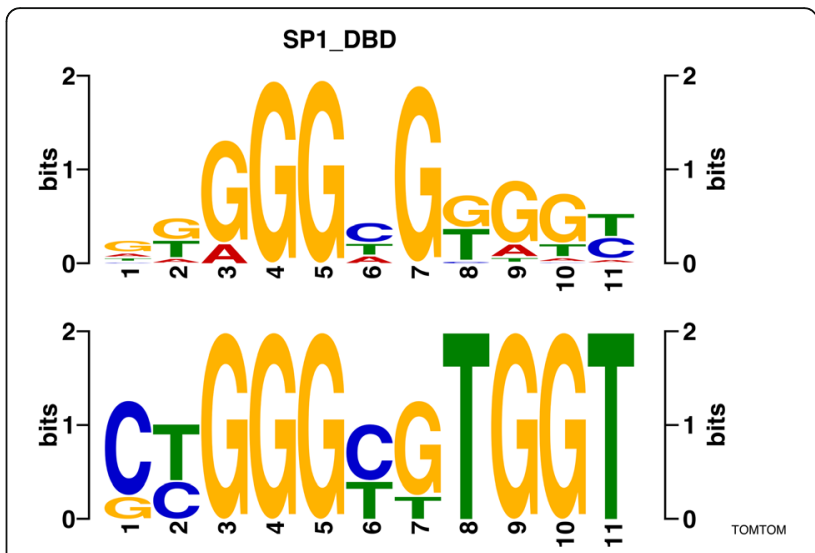

Fig. 4 MEME-ChIP [38] enriched sequence identified in hypomethylated a-DMRs (below) and TOMTOM (v4.10.2) enrichment for the SP1 transcription factor motif (above)

Next, to check for blood-cell type bias we performed six epigenome-wide association studies (EWAS) in 54 monozygotic (MZ) discordant twins with precise white blood cell data for $\mathrm{CD}_{4}^{+}$helper $\mathrm{T}$; $\mathrm{CD}^{+}$cytotoxic $\mathrm{T}$; $\mathrm{T}$ cell; natural killer cell; $\mathrm{CD} 34^{+}$multipotential haematopoietic stem cell; and B cells [44] within the entire DNA methylome dataset. In this $\mathrm{MZ}$ analysis, $6.44 \%$ of all windows have $p<0.05$ for any of these six blood traits. However, a slightly lesser value of $6.38 \%$ have a $p<0.05$, and none near Bonferroni, within the a-DMR windows. Therefore, there is no strong evidence for enrichment for blood-cell changes in the a-DMRs $\left(\chi^{2} p>0.05\right)$.

\section{Validation of a-DMRs}

In a dataset of 811 individuals that possessed $450 \mathrm{k}$ array blood data (mean age, 58.0 years; age range, $18.6-81.7$ years; $88.9 \%$ overlap with MeDIP samples), we attempted to validate the fraction of a-DMRs that had overlapping probe(s). Thirty-eight a-DMRs possess at least one overlapping $450 \mathrm{k}$ probe and, of these, $36 \mathrm{a}-\mathrm{DMRs}$ included probe(s) that passed quality control (QC). We performed a similar linear mixed effect model analysis for ageing methylation changes including the same covariant information as in our MEDIP-seq data. In this investigation, 32 (88.9 \%) and 25 (69.4\%) of these 36 a-DMRs had at least one CpG with a nominal or Bonferroni significant $\left(p<1 \times 10^{-7}\right)$ results with the same directional change, respectively (Additional file 2 : Table S3). This thus strongly supports our results but also shows the unique power of our analysis.

Of note, those a-DMRs that did possess an overlapping probe(s) were almost exclusively those where methylation increases with age, 34 of the total 38 and 31 of the 32 with at least nominally significant probes. This is thus consistent with early array studies that predominately found this direction of change, but also starkly revealing the bias of the CpGs present on the array. 


\section{Replication of a-DMRs}

The significant windows of the 71 a-DMR loci were assessed in a non-overlapping dataset of 2084 DNA methylomes also sourced from TwinsUK. This analysis was performed identically as for the discovery set, but with reduced covariate information for the genotype, smoking or leukocyte categories. Of the total number of 115 Bonferroni significant ageing-related windows, $96.5 \%$ (111) show the same direction of effect, $84.3 \%$ (97) nominal significance $(p<0.05)$ and $60.9 \%(70)$ are even significant beyond the discovery Bonferroni level $\left(p<1.85 \times 10^{-8}\right)$. Of the merged 71 a-DMRs, $68(95.7 \%)$ have consistent windows with the same direction of effect, $57(80.3 \%)$ include a window that is nominally significant $(p<0.05)$ and in $38(53.5 \%)$, this reaches Bonferroni significance in this replication dataset $(p<$ $1.85 \times 10^{-8}$; Additional file 2: Table S1).

\section{Individual a-DMR loci}

We identified numerous a-DMRs in genetic regions with intriguing additional evidence from the literature for potential roles in age-related phenotypes. A selection of these a-DMR results are discussed below and are shown in Fig. 5, Additional file 3: Figure S1, and all in Additional file 4: Figure S2. The top a-DMR overlapped an intragenic 3' CpG island and shore region within the HSPA2 (Heat Shock 70 kDa Protein 2) gene (chr14:65,008,750$65,009,500)$, with the peak window $p=7.14 \times 10^{-21}$. It is within a GM12878 ChromHMM predicted poised promoter and possesses strong vertebrate conservation (Fig. 5a). This precise locus was also previously identified in the MBD-seq study by McClay et al. [12] and also in a paediatric cohort via the $27 \mathrm{k}$ array [5].

A number of physiologically interesting genetic loci are associated with the a-DMRs. This includes novel DNA hypomethylation in the IGFBP4 promoter, with previously identified ageing-related increases in this gene product in both human serum and bone and a proposed role in bone loss in the elderly [45] (Fig. 5b). Another novel finding was within an intragenic a-DMR within $C D C 14 B$, residing over multiple-tissue enhancer predictions (Additional file 3: Figure S1c). A Cdc14b knockout mouse model displays an early ageing phenotype and a defective DNA damage response [46]. Murine data also shows enhancer evidence in this location and conserved epigenomic enhancer signals have been shown to be highly informative in disease models [47].

An intragenic a-DMR within HFE, the Haemachromatosis gene, resides upstream of the two common nonsynonymous SNPs causative in this autosomal recessive condition (Additional file 3: Figure S1d). This is a low penetrance age-related phenotype with the symptoms only usually appearing between 30 and 50 years. The aDMR overlaps predicted weak enhancer signal in hepatocellular-derived HepG2 ChromHMM data. If this novel change is occurring across additional tissues, such

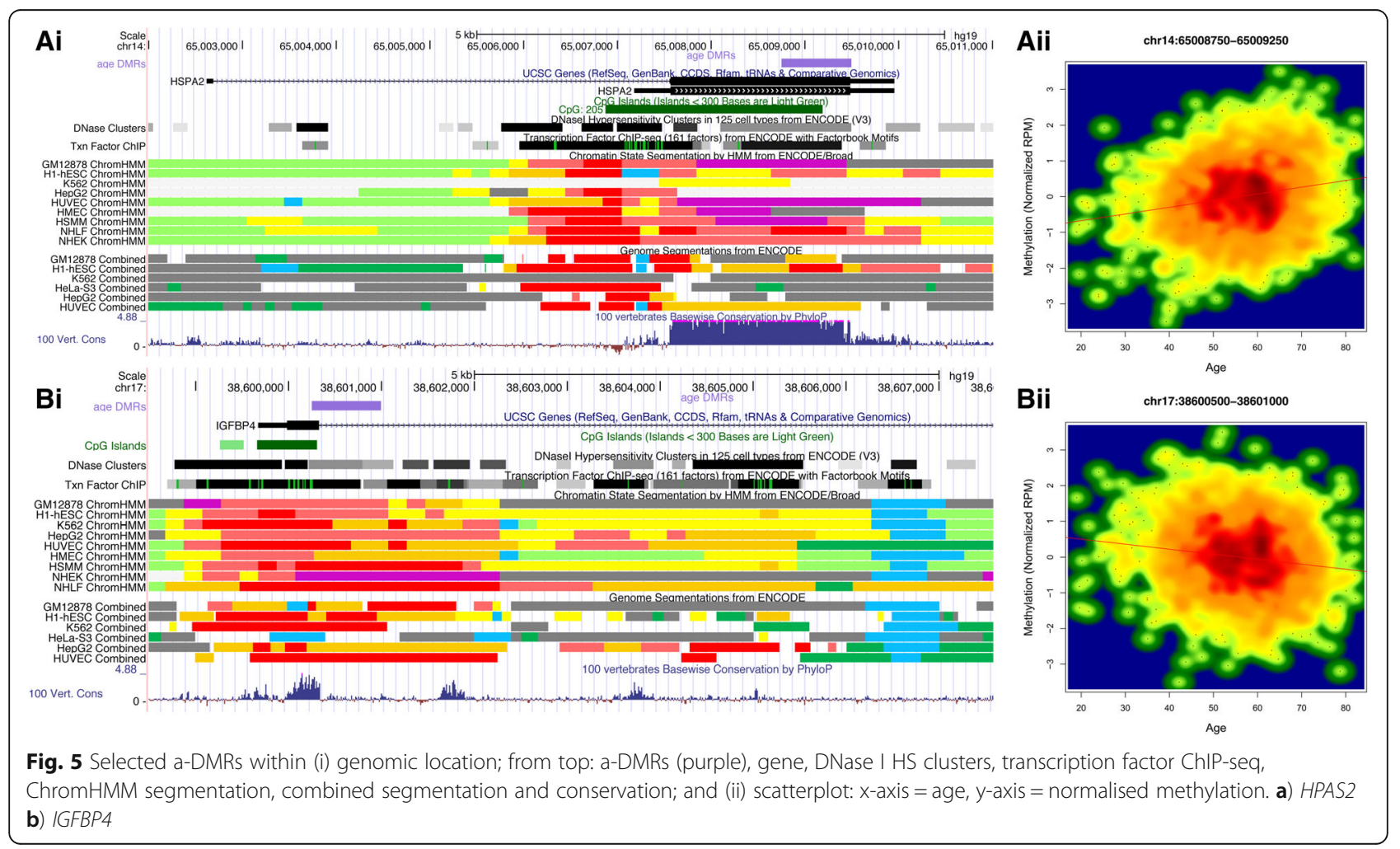


as the liver, it may be involved in the pathophysiology. However, there is no current array coverage with the nearest probe $>\sim 1 \mathrm{~kb}$ further upstream away. Located in the gene body within the $0.3-8 \mathrm{~kb}$ downstream region of the promoter, it would also be defined as an undefined intragenic DMR (uiDMR), as per Schultz et al., shown to strongly influence expression [48].

Two a-DMRs reside within the vicinity of the NR4A2 gene, one within an intragenic CpG Island shore and the other $\sim 1.5 \mathrm{~kb}$ downstream from this gene, both overlapping predicted poised promoters (Additional file 3: Figure S1e). This gene itself is associated with nutritional status, postnatal development and hormonal imbalances [49]. Another a-DMR resides in the poised promoter of the growth hormone secretoagogue receptor (GHSR), or Ghrelin receptor, for the orexigenic hormone ghrelin that is active in the hypothalamus (Additional file 3: Figure S1f) with potential age-related effects [50]. Additional novel a-DMRs in genes of interest include BMI1, with an associated brain ageing phenotype in mouse knockout [51], and C14orf39, with a non-synonymous SNP associated with menarche [52].

Earlier studies have found numerous ageing changes within cancer-related genes and we also find many genes in this category, including previously identified loci in CDKN2A (also with a well-known role in ageing [53]), $M G A$ and ZNF577, and novel changes in ZNF300P1 (Additional file 3: Figure S1g), STEAP1, FOXE1 and $P A X 1$. Four overlapping significant windows comprise the $1.25 \mathrm{~kb}$ a-DMR in ZNF577 that completely overlaps its $5^{\prime}$ promoter $\mathrm{CpG}$ island (Additional file 3: Figure S1h). This CpG island is known to be hypermethylated in lung and other cancers, but of particular interest to this blood tissue study, it was also found to be the only gene with promoter hypermethylation in a study of polycythaemia vera (PCV) JAK2 V617F mutation individuals [54]. PCV is usually a late onset disease that is commonly diagnosed around 60-65 years. Subclinical PCV with undetectable low-level $J A K 2$ somatic clonal mutations could potentially contribute to this signature within this ageing cohort. This a-DMR location also overlaps with three previous studies $[6,8,11]$.

All the above-mentioned genes were significant beyond the Bonferroni level in the replication set, except for C14orf39 (replication $p=2.953 \times 10^{-6}$ ).

\section{Multi-tissue regulatory enrichment}

We investigated the a-DMRs to ascertain whether they were more likely to fall within DHSs identified within blood-cell tissues and/or other cell types. This revealed that while the a-DMRs are enriched within this regulatory marker in blood cells, they were in fact significant across all tissue types and not particularly within haematological tissue (125 ENCODE DHS tissue types [55], all $\chi^{2} p<2.2 \times 10^{-16}$; Fig. 6). This implies their systemic functional potential. Those that do reside within blood are an interesting subset due to their direct mechanistic interpretation. Twelve a-DMRs show a strong enrichment in blood-related DHS ( $\geq 50 \%$ of the 22 blood-cell related analyses out of 125 total, Additional file 2: Table S4). These include novel a-DMRs within the promoter of TAOK2 involved in the MAPK signalling pathway implicated in degenerative disease [56] (Additional file 3: Figure S1i) and within the promoter of an isoform of FADS2 associated with liver omega desaturation [57]. a-DMRs that overlapped with previous studies include: an intragenic region within $A F F 1$, the 3' portion of the oncogenic fusion gene causative in acute lymphoblastic leukaemia [58] (Additional file 3: Figure $\mathrm{S1j}$ ); the promoter CpG Island shore region of the transcription factor KLF9 induced by oxidative stress [59]; and the ZNF577 a-DMR mentioned above with respect to PCV.

\section{a-DMRs that reside within blood-related disease regions} Age-related DNA methylation changes within GWAS disease-associated regions where blood tissue may be directly involved in the pathophysiology make strong candidates for an epigenetic ageing and genetic interaction. The 71 a-DMRs reside within 64 GWAS LD blocks, containing 242 unique SNPs that have been related to 534 overlapping diseases and traits (Additional file 2: Table S5). When categorised by 15 broad disease classes [60], we found associations for haematological measures (24 associations), autoimmune-related diseases (34) and large numbers of associations across an overlapping range of serum metabolites (257). All of these may influence or could be represented in blood physiological changes (Additional file 2: Table S6). In fact, a large proportion of the a-DMRs $(33, \sim 46.48 \%$ ) are implicated in these three broad disease categories. Additionally, within the cancer category, there are two blood-related malignancies with a-DMRs within their GWAS association risk loci: multiple myeloma and acute lymphoblastic leukaemia.

\section{Age-related disease enrichment}

We additionally parsed our a-DMR results for an enrichment for ageing-related diseases. While many disorders and phenotypes have some complex age component, we restricted this analysis to adult-onset cancers, agerelated and degenerative neurological, musculoskeletal, metabolic, cardiac and cerebrovascular diseases, as well as cognitive decline and age-of-onset analyses (Additional file 2: Table S7). This revealed an enrichment for the aDMRs to reside within GWAS LD blocks for an agerelated phenotype. These disorders are found in $26.7 \%$ of the GWAS LD blocks, compared to $39.4 \%$ of the a-DMR co-locating GWAS LD blocks, Fisher's exact test $p=0.02$, 


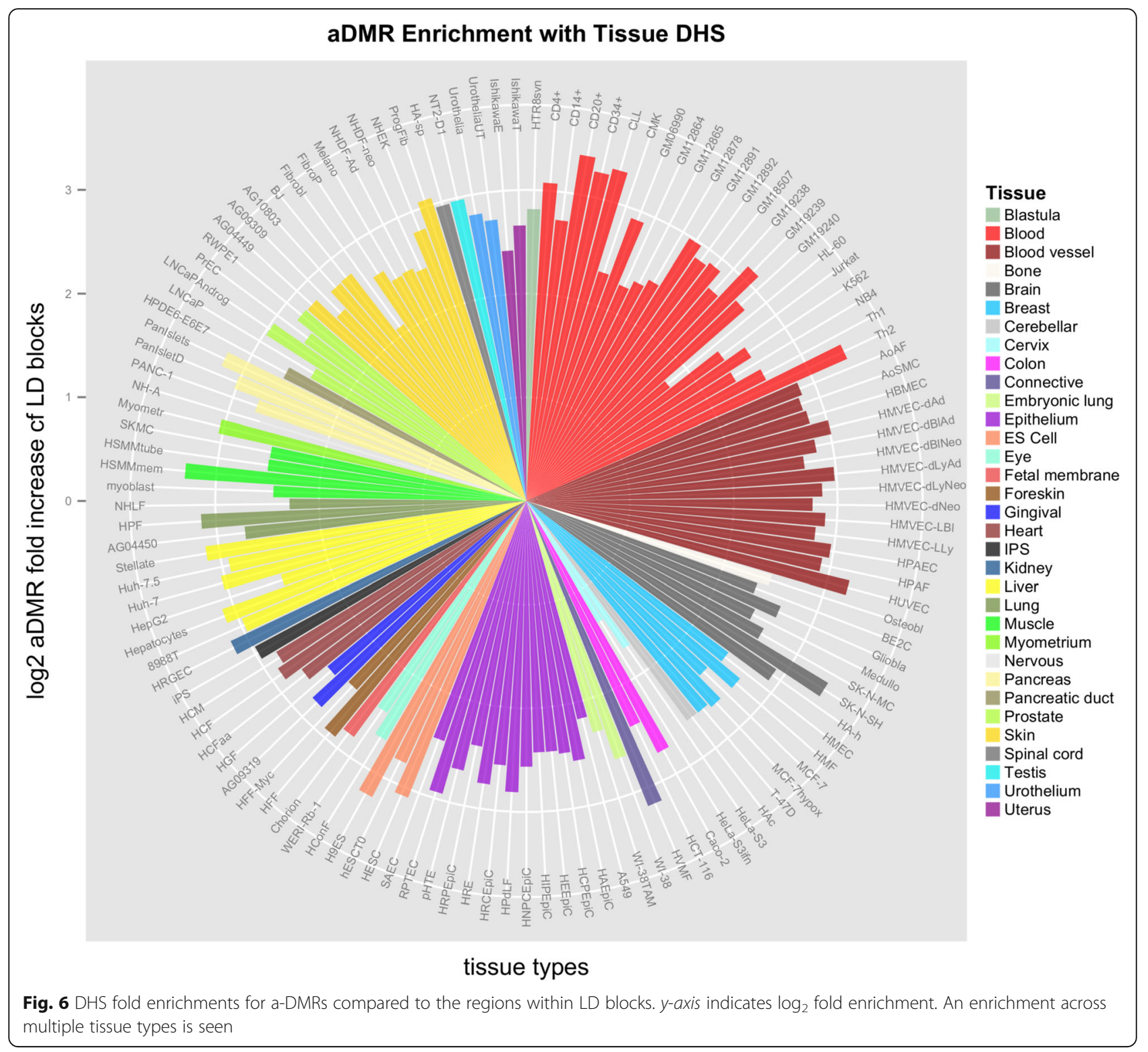

$\mathrm{OR}=1.81$, CI 1.08-3.02). Permutation analysis, by randomly selecting 1000 times the age-related fraction of GWAS LD blocks from the total set and then testing overlap with the observed a-DMRs, significantly supported this enrichment (empirical $p$ value $=0.021$ ).

\section{Interaction with genotype}

While genetic effects can strongly confound an EWAS analysis, we attempted to explore our dataset for any evidence of a genetic facilitated effect, through common haplotype differences, on DNA methylation changes occurring with age. We performed an interactive analysis between the LD block haplotype tagging genotype and age with DNA methylation. We then overlapped these results with the a-DMR regions, where we had excluded genotype being the sole driver of the signal. Thus we are not looking for obligatory differences, but instead an accentuated facilitated ageing signature on either the risk or non-risk haplotype, with the potential to then have influence on the associated phenotype or disease.

Three a-DMRs were identified that co-located with significant interaction results (all $p<0.05$, Additional file 5: Figure $\mathrm{S} 3 \mathrm{~A}-\mathrm{C}$ ). One of these included the enhancer located a-DMR in the TRAK1 locus associated with the late onset neoplasm, multiple myeloma (rs1052501). Other SNPs within this LD block associate with additional phenotypes, such as blood pressure. The $\mathrm{G}$ risk allele carriers did not show the same increase in DNA methylation with age, possibly indicating that these individuals have a prematurely higher DNA methylation in this locus and therefore the age-related trend is not seen. The a-DMR within the promoter of the MGA promoter 
also shows this trend, with the SNP rs28374715 in this LD block associated with ulcerative colitis. The a-DMR within the promoter of the IGFBP4 gene, previously mentioned with regards to age-related bone loss, shows methylation change in those individuals with the SNP rs584438 T allele, which is related to height, decreasing more strongly than the non-associated haplotype.

\section{Discussion}

Epigenomic changes with advancing years provide a novel avenue to explore the physiology and mechanisms of ageing [9]. This is clear with the observed discordance between chronological and predicted epigenetic age and its association with mortality [18]. The driving role of heterochromatic epigenetic changes in ageing has also been displayed in the human premature ageing disorder Werner syndrome [61]. In this study, we performed the largest analysis of DNA methylation changes with age to date and identified novel age-related regions that show strong functional enrichment over a range of publicly available evidence. In comparison to previous array findings, in almost half of these loci DNA methylation decreased with age. Those a-DMRs that did overlap array probes were almost exclusively regions that hypermethylate with age, clearly displaying the bias of the array to $\mathrm{CpGs}$ possessing this directional change only. The identification of these novel ageing changes in loci with no array coverage reinforces the insights more genome-wide methodology can provide. Furthermore, additional evidence that strong associations are identified in this study comes from the convincing replication in an independent large dataset.

The a-DMR loci co-locate with many physiologically interesting genes, including novel and robustly replicated findings in IGFBP4, CDC14B, HFE, BMI1 and TAOK2, among others. These loci reside within regions that have strong genetic associations with common diseases and therefore are novel candidates for potential genetic and epigenetic interactions, particularly for late-onset conditions. Blood-derived DNA methylation ageing changes can be concordant across a range of other tissues $[9,15]$, consequently some of these alterations may act in the most disease-relevant tissue. We identified that our ageing changes were enriched for the regulatory regions of multiple tissue types. Furthermore, epigenetic variation is strongly intertwined with sequence-specific transcription factor interaction and binding $[62,63]$ and thus these changes can be a key to unlocking the precise molecular mechanisms involved.

Multiple cell-types are present in peripheral blood, therefore its analysis represents a meta-epigenome [64], so that changes in subpopulation proportions will be detected as epigenetic variation. However, we found no strong evidence of enrichment for blood cell-type related DNA methylation changes within these a-DMRs. Additionally, contribution to DNA methylome variation by subclinical or unknown pathology, as identified in recent age-related brain analysis via neuropathologies [65], cannot be completely excluded. Although this is unlikely to be present in our samples, since TwinsUK participants are recruited as predominately healthy volunteers, unselected for any diseases.

There are inherent power benefits from this study that is six-fold larger in sample size to the only comparable previous analysis for regional changes by McClay et al. [12]. Robust DMRs are strongly enriched for function [66]; however, denser coverage is required for successful DMR calling than is provided by $450 \mathrm{k}$ array [67]. Fundamentally, arrays are designed to identify individual CpGs in contrast to regional approaches, such as MeDIP-seq and MBD-seq, which can only identify consistent regional variation. Thus while the 71 a-DMRs may not initially compare to multiple a-DMPs identified with arrays, this is inherent with these methodical differences. Our results are directly analogous to the 11 aDMRs identified in the other large-scale genome-wide approach of McClay et al. [12].

The finding in this study of aberrant DNA methylation within disease-related loci proposes potential interrelationships with ageing changes and disease susceptibility or downstream consequence. Evidence that age-associated DNA methylation may predispose to cancer by reducing threshold of malignant transformation has been previously shown [68] and is implicated in the increased cancer incidence with age [15]. The ability of epimutations to pathogenically drive disease in the same fashion as genetic mutation, such as in acute myeloid leukaemia [69], further support the pathological potential of these changes and also the possibilities in regards to nonmalignant disease [70].

\section{Conclusion}

The loci we have investigated are associated with human traits and disease through robust and replicated GWAS. Thus these epigenetic changes with age will be valuable measures to incorporate in these disease models. With the availability of genotype data, we have also been able to test and identify variation in this signal between risk and nonrisk haplotypes. With a more detailed understanding of the haplotypic nature of both genetic risk [71, 72], but also epigenetic risk, through the integration of obligatory and facilitated epigenetic changes [27, 73, 74], a more precise understanding of common disease will emerge. This welldefined allele-specific genetic and epigenetic variability should accelerate mechanistic discoveries into ageing's role in late-onset disorders and the biology of human ageing and disease. 


\section{Methods}

\section{Participants}

Participants are from the deeply phenotyped UK Adult Twin Register (TwinsUK Resource) [75] based at St Thomas' Hospital, London. Phenotyping occurs at interview when blood is also taken for haematological analysis and DNA extraction. Storage is in EDTA tubes at $-80{ }^{\circ} \mathrm{C}$. Nucleon Genomic DNA Extraction Kits are used for DNA extraction which are then stored at $-20{ }^{\circ} \mathrm{C}$ in TE buffer. Haematological analysis for full blood count was performed on the majority of extracted bloods. Smoking status is recorded at this time or within the nearest five years via questionnaire if not available. Zygosity is determined by twinning questionnaire and confirmed by genotyping.

The discovery set consisted of 2238 DNA methylomes, which were all female, therefore sex-specific modifications were removed [76], and included longitudinal data with two or more time points on 408 individuals (mean time difference 2.18 years) and single time point data on 1350. These 1758 individuals included $203 \mathrm{MZ}$ twin pairs and $489 \mathrm{MZ}$ singletons and 371 dizygotic (DZ) pairs and $121 \mathrm{DZ}$ singletons, therefore comprising equal numbers of $\mathrm{MZ}$ (50.9 \%) and DZ (49.1 \%) individuals from a total of 1184 unique families. The age at collection date of blood for DNA extraction was in the range of 19-82.2 years (mean age, 55.99 years; median age, 56.60 years; std. dev. 10.32 years).

\section{MeDIP-seq}

DNA sample preparation, MeDIP reaction and Illumina second-generation sequencing were all performed at BGI-Shenzhen, Shenzhen, China. Fragmentation of the whole peripheral blood TwinsUK DNA was via sonication with a Covaris system (Woburn, MA, USA). Libraries for sequencing were prepared from 5 ug of fragmented genomic DNA. End repair, $<\mathrm{A}>$ base addition and adaptor ligation steps were performed using Illumina's DNA Sample Prep kit for single-end sequencing. The anti-5mC antibody (Diagenode) was used to immunoprecipitate the adaptor-ligated DNA and the resultant MeDIP was validated by quantitative polymerase chain reaction (PCR). This captured DNA was then purified with Zymo DNA Clean \& Concentrator $^{\mathrm{Tm}}-5$ (Zymo Research) and subsequently amplified with adaptor-mediated PCR. Fragments of size 200-500 bp were selected by gel excision and then $\mathrm{QC}$ assessed by Agilent BioAnalyzer analysis. These libraries were then sequenced on the Illumina platform. Sequencing data passed initial QC for base composition assessed via FASTQC (v0.10.0) (http://www.bioinformatics.bbsrc.ac.uk/projects/fastqc). MeDIP-seq data were processed with BWA (Burrows-Wheeler Aligner) alignment [77] (passing a mapping quality score of Q10), with duplicates removal, FastQC and SAMTools [78] QC and MEDIPS(v1.0) [79] for MeDIP-specific analysis, QC, reads per million (RPM) and absolute methylation score (AMS) generation. The average high quality BWA aligned reads was $\sim 16.9$ million per sample for the discovery set of 2238 and $\sim 16.8$ million for the replication set of 2084. Further QC was performed via R (correlation matrix, hierarchical clustering, dendogram, heatmap, density plot) and batch effects inspection by principle component analysis. Processed data for statistical analysis are BED files of genomic windows (500-bp, 250-bp slide) with RPM scores. All human genome coordinates, calculations performed and those cited are in build hg19/GRCh37.

\section{GWAS LD blocks}

The analysis was performed on the a priori functionally enriched genomic regions contained within the LD blocks of the NIH GWAS SNP catalogue [24, 25]. The LD blocks were ascertained from the GRCh37 genetic map, downloaded from Center of Statistic Genetics, University of Michigan, Locuszoom 1.3 [80], with recombination rate of $10 \mathrm{cM} / \mathrm{Mb}$ boundaries. LD blocks were further pruned to those $\leq 10 \mathrm{Mb}$ in size. We selected the 8093 curated GWAS SNPs with $p$ value $<1 \times 10^{-7}$ deposited within the NIH GWAS catalogue as at December 2014. Due to co-associations for the same SNP, these are 5522 unique individual SNPs and 5477 of these resided within the above-identified LD blocks. In fact, these represented 2709 distinct LD blocks once accounting for SNPs present within the same block. These regions cover $\sim 22.1 \%$ of the human genome.

\section{Age-associated DNA methylation analysis}

All statistical analyses were run in the $\mathrm{R}$ (3.0.0) environment [81]. The lme4 package [82] was employed to perform a linear mixed effect analysis of the relationship between chronological age at DNA extraction and DNA methylation, which was represented as normalised RPM values within the 500-bp windows. Additional fixed effects terms included allelic count of the haplotypetagging SNP, smoking status, batch, blood cell subtypes (lymphocytes, monocyte, neutrophil and eosinophil) with family and zygosity as random effects. This model for DNA methylation age analysis is similar to that used previously in array based analyses [15] with the additional inclusion of genetic allelic information. $p$ values were calculated with the ANOVA function by likelihood ratio test of the full model including age versus null model excluding this variable. A Bonferroni multiple testing correction was calculated by the total number of DNA methylation windows included in the analysis $(2,708,462)$, giving a $p$ value significance level of $<1.85 \times 10^{-8}$ (see "Study Design" in Additional file 6: Figure S4). 
The immunoprecipitation reaction in MeDIP-seq data is extremely susceptible to the influence of genetic variation in CpG number (due to CpG-SNPs, CNVs, indels and STRs), leading to a direct relationship between the number of methylated cytosines in the DNA fragment and the amount of DNA captured by the antibody as discussed by Okitsu and Hsieh [22]. We accounted for this influence by the inclusion of the haplotype-tagging common SNP data for each LD block examined within our statistical model. We further also removed the ENCODE poor mappability blacklist regions [28] from any further analysis (13,726 500-bp windows). Shared trans factors, however, cannot be accounted for, although are much less frequent [83], but the large replication set, described below, adds powerful support to the discovery findings.

An interaction between genotype and age was directly tested for by comparing the full model, but with DNA methylation and age included as interacting factors, and the full model in the initial analysis, with again a likelihood ratio test via ANOVA to derive significance levels. As the direct confounding of common genetic effects was included in the initial a-DMR analysis with strict Bonferroni cutoff, we then overlapped these results with our a-DMR set to identify those robust a-DMRs with potential evidence of interaction.

\section{Novelty of a-DMRs analysis}

We identified 14 previous studies [3-16] that had been performed for DNA methylation changes in blood with respect to age with available data for comparison and downloaded these results placing CG positions at their correct co-ordinates from Illumina array annotation files and converting all that were in previous builds to hg19/ GRCh37 via UCSC tools liftOver [84]. These were merged and compared via BEDtools (v.2.17.0) and are available in Additional file 7.

\section{Blood-cell discordant monozygotic twin EWAS}

A MZ discordant EWAS in $54 \mathrm{MZ}$ pairs that possessed precise white blood cell data within this DNA methylome dataset was performed. These data were generated by Roederer et al. [44] and included calculations for $\mathrm{CD}^{+}$helper $\mathrm{T}, \mathrm{CD}^{+}$cytotoxic $\mathrm{T}, \mathrm{T}$ cell, natural killer cell, CD $34^{+}$multipotential haematopoietic stem cell and B cells. MZ twin pairs' discordance for each blood-cell trait was calculated. The 500-bp DNA methylome windows for analysis required $\geq 90 \%$ of individuals with non-zero values. Residuals from the linear regression model of RPM methylation scores with adjustments for smoking, leukocyte counts, age at DNA extraction and batch were normalised (qqnorm) and then the high-low difference significance was compared by one-sided T-test.

\section{Enrichment analysis}

Initial exploration of a-DMRs was performed via Epiexplorer [85]. This enabled enrichment for chromatin state (ChromHMM), histone modifications and additional ENCODE and Roadmap data to be investigated first. Comparisons were made with ChromHMM in nine tissues from Encode Broad HMM (Gm12878; H1hesc; Hepg2; Hmec; Hsmm; Huvec; K562; Nhek; Nhlf) and then with combined segmentation in six tissues - Encode AwgSegmentation (Gm12878; H1hesc; Helas3; Hepg2; Huvec; K562) via UCSC. Overlap in genetic and functional data was calculated with BEDtools (v.2.17.0) command intersectBed, compared with non-overlapping LD block 500-bp windows with -f 0.1 parameter (moderate overlap). The genetic regions compared for enrichment were CpG islands, TFBSs from ENCODE v3 (690 datasets from wgEncodeRegTfbsClusteredV3 [86]), DHS in 125 cell types from ENCODE analysis [55] and Vertebrate Multiz Alignment and Conservation (100 Species) from 100Vert_El_phastConsElement100way bedfile ( $10.1 \mathrm{~m}$ regions), all downloaded from UCSC [87]. FANTOM5 enhancers regions were from Anderson et al. [36] and 'Dynamic' regions from Ziller et al. [66].

A further a-DMR enrichment analysis was performed with the Genomic Regions Enrichment of Annotations Tool (GREAT v3.0.0) [88] region-based binomial analysis with basal, but the extension parameters reduced from the default (constitutive $5.0 \mathrm{~kb}$ upstream, $1.0 \mathrm{~kb}$ downstream and up to $100 \mathrm{~kb}$ max extension, not $1 \mathrm{Mb}$ ). $\mathrm{Cu}$ rated regulatory domains were included and all LD block regions were used as the background set.

For TFBS motif enrichment, we used the TRAP method [37] and the MEME suit (MEME-ChIP [38] and TOMTOM (v4.10.2) [89]). FASTA sequence files of the 71 a-DMRs were inputted as separated hypomethylated and hypermethylated groups. In TRAP they were compared to the JASPAR vertebrates with a background model of human promoters. MEME-Chip compared with a set of 1229 DNA motifs, in the range of 7-23 in length (average length 13.8), from the database Human and Mouse (in silico).

\section{Validation analysis}

Within the a-DMRs, 116 CpG probes from the Infinium Human Methylation450 BeadChip reside that passed QC, as detailed below. These were blood-derived CpG methylation scores from 811 female individuals, $89.1 \%$ overlapped with the MeDIP samples. QC included removal of probes that failed detection in at least one sample and with a bead count less than 3 in more than $5 \%$ of the samples, and probes for which the 50 bp sequence aligned to multiple locations in the genome. Cell type proportions were estimated for CD8+ T cells, CD4+ T cells, B cells, natural killer cells, granulocytes and monocytes [43]. All 
data were normalised using the intra-array normalisation, beta-mixture quantile dilation (BMIQ) [90] to correct for probe type bias. The validation was performed using a linear mixed effects model fitted on standardised beta values per probe $(\mathrm{N}(0,1))$ with age, genotype as allelic count, smoking status, beadchip, position on the beadchip, granulocytes, monocytes and CD8+ T cells as fixed effects, as well as family and zygosity as random effects. To assess for significance, ANOVA was used to compare this model to a null model without age.

\section{Replication analysis}

We utilised an additional 2084 peripheral blood MeDIPseq data, also available from TwinsUK, for our replication set. None of these individuals were present in the discovery set and do not differ from that set in any selective way. These samples were in the age range of 16-82.2 years (mean age, 51.00 years; median age, 53.40 years; std. dev. 14.91), were $87.04 \%$ female and included 1897 samples from $1710 \mathrm{MZ}$ individuals (582 pairs, 546 lone) and 187 samples from 159 DZ individuals (46 pairs, 67 lone), with 215 possessing data from $>1$ time point. Analysis was performed as for the discovery set using an identical linear mixed effect model, for normalised DNA methylation (500 bp windows) with age at DNA collection; however, these samples did not possess genotype, smoking or leukocyte information, and therefore only included the additional fixed effect of batch and random effects of zygosity and family.

\section{Tissue-specific investigation}

The DHS from 125 cell type experiments from ENCODE analysis [55] were used for tissue-specific analysis of the a-DMRs. This dataset includes 22 blood tissue related samples. Broad disease classes were taken from Maurano et al. [60].

\section{Additional files}

Additional file 1: BED file of a-DMRs with UCSC browser header.
(BED $1 \mathrm{~kb}$ )

Additional file 2: Supplementary Tables S1-7. (XLSX 1125 kb)

Additional file 3: Figure S1. Selected a-DMRs within (i) Genomic Location; From Top: a-DMRs (purple), Gene, DNase I HS Clusters, Transcription Factor ChIPseq, ChromHMM Segmentation, Combined Segmentation, \& Conservation. (ii) Scatterplot: $X$ axis $=$ Age, $Y$ axis $=$ Normalised Methylation. C) CDC14B, D) HFE, E) NR4A2, F) GHSR, G) ZNF300P1, H) ZNF577, I) TAOK2, J) AFF1 locus. (PDF 5.8 mb)

Additional file 4: Figure S2. All a-DMRs within (1) genomic location. Top: a-DMRs (purple), gene, DHS clusters, transcription factor ChIP-seq, ChromHMM segmentation, combined segmentation and conservation; and (2) scatterplot: $x$-axis $=$ Age, $y$-axis $=$ Normalised methylation. (PDF 34909 kb)

Additional file 5: Figure S3. Genotype-interaction analysis. Variation in ageing signal depending on genotype. Homozygote SNP trait related allele is in red. Left: Scatterplot: $x$-axis $=$ Age, $y$-axis $=$ Normalised methylation. Right: Boxplot: three genotype categories separated into two groups by $\leq$ or $>$ median age (56.6 years). (PDF $910 \mathrm{~kb}$ )

Additional file 6: Figure S4. Study design flowchart. (PDF $152 \mathrm{~kb}$ ) Additional file 7: BED file of combined 14 ageing studies. (BED $2508 \mathrm{~kb}$ )

\section{Abbreviations}

a-DMR: Ageing-related differentially methylated region; CGI: CpG island; DMP: Differentially methylated position; DMR: Differentially methylated region; EWAS: Epigenome-wide association study; GWAS: Genome-wide association study; LD: Linkage disequilibrium; MeDIP-seq: Methylated DNA immunoprecipitation second-generation sequencing; WGBS: Whole genome shotgun bisulfite second-generation sequencing

\section{Funding}

Funding support for this project was obtained from the European Research Council (project number 250157) and BGI. TwinsUK received funding from the Wellcome Trust; European Community's Seventh Framework Programme (FP7/2007-2013), the National Institute for Health Research (NIHR)-funded BioResource, Clinical Research Facility and Biomedical Research Centre based at Guy's and St Thomas' NHS Foundation Trust in partnership with King's College London. SNP genotyping was performed by The Wellcome Trust Sanger Institute and National Eye Institute via NIH/CIDR. CGB receives support from the MRC Lifecourse Epidemiology Unit and the EpiGen Global Research Consortium (www.epigengrc.com). TDS is holder of an ERC Advanced Principal Investigator award.

\section{Availability of data and materials}

The data for this article and the long-term data storage for the EPITWIN DNA methylome project are in the European Genome-phenome Archive (EGA), coordinated by the European Bioinformatics Institute (EBI) and the Centre for Genomic Regulation (CRG) (https://www.ebi.ac.uk/ega). It is deposited under study number EGAS00001001910 and dataset EGAD00010000983.

\section{Authors' contributions}

CGB devised the LD-aware MeDIP-seq DNA methylome ageing study and analysed and interpreted the results. TDS, JB, KW and JW conceived and designed the experiments to produce the MeDIP-seq dataset. YX, FG and JW produced and WY and JB processed and quality controlled these data. MM and PGH contributed additional genetic data on these TwinsUK individuals for this analysis. LR contributed data quality control and analysis of the $450 \mathrm{k}$ validation dataset. CGB, with input from TDS and PGH, wrote the paper. All authors reviewed and approved the final manuscript.

\section{Competing interests}

The authors declare that they have no competing interests.

\section{Ethics approval and consent to participate}

Written informed consent from all participants was obtained in accordance with Guy's \& St Thomas' NHS Foundation Trust Ethics Committee (EC04/ 015 - 15-Mar-04). Experimental methods comply with the guidelines of the Declaration of Helsinki.

\section{Author details}

${ }^{1}$ Department of Twin Research \& Genetic Epidemiology, King's College London, London, UK. ${ }^{2}$ MRC Lifecourse Epidemiology Unit, University of Southampton, Southampton, UK. ${ }^{3}$ Human Development and Health Academic Unit, Institute of Developmental Sciences, University of Southampton, Southampton, UK. ${ }^{4}$ Epigenomic Medicine, Biological Sciences, Faculty of Environmental and Natural Sciences, University of Southampton, Southampton, UK. ${ }^{5}$ BGI-Shenzhen, Shenzhen 518083, China. ${ }^{6}$ Institute of Cancer Research, Sutton, UK.

Received: 17 May 2016 Accepted: 31 August 2016 Published online: 23 September 2016

References

1. Valdes AM, Glass D, Spector TD. Omics technologies and the study of human ageing. Nat Rev Genet. 2013;14:601-7. 
2. Fraga MF, Ballestar E, Paz MF, Ropero S, Setien F, Ballestar ML, et al. Epigenetic differences arise during the lifetime of monozygotic twins. Proc Natl Acad Sci U S A. 2005;102:10604-9.

3. Teschendorff AE, Menon U, Gentry-Maharaj A, Ramus SJ, Weisenberger DJ, Shen $\mathrm{H}$, et al. Age-dependent DNA methylation of genes that are suppressed in stem cells is a hallmark of cancer. Genome Res. 2010;20:440-6.

4. Rakyan VK, Down TA, Maslau S, Andrew T, Yang TP, Beyan H, et al. Human aging-associated DNA hypermethylation occurs preferentially at bivalent chromatin domains. Genome Res. 2010;20:434-9.

5. Alisch RS, Barwick BG, Chopra P, Myrick LK, Satten GA, Conneely KN, et al. Age-associated DNA methylation in pediatric populations. Genome Res. 2012;22:623-32.

6. Heyn H, Li N, Ferreira HJ, Moran S, Pisano DG, Gomez A, et al. Distinct DNA methylomes of newborns and centenarians. Proc Natl Acad Sci U S A. 2012; 109:10522-7.

7. Garagnani P, Bacalini MG, Pirazzini C, Gori D, Giuliani C, Mari D, et al. Methylation of ELOVL2 gene as a new epigenetic marker of age. Aging Cell. 2012;11:1132-4.

8. Bell JT, Tsai PC, Yang TP, Pidsley R, Nisbet J, Glass D, et al. Epigenome-wide scans identify differentially methylated regions for age and age-related phenotypes in a healthy ageing population. PLoS Genet. 2012;8, e1002629.

9. Horvath S. DNA methylation age of human tissues and cell types. Genome Biol. 2013;14:R115.

10. Hannum G, Guinney J, Zhao L, Zhang L, Hughes G, Sadda S, et al. Genomewide methylation profiles reveal quantitative views of human aging rates. Mol Cell. 2013:49:359-67.

11. Day K, Waite LL, Thalacker-Mercer A, West A, Bamman MM, Brooks JD, et al. Differential DNA methylation with age displays both common and dynamic features across human tissues that are influenced by CpG landscape. Genome Biol. 2013:14:R102.

12. McClay JL, Aberg KA, Clark SL, Nerella S, Kumar G, Xie LY, et al. A methylome-wide study of aging using massively parallel sequencing of the methyl-CpG-enriched genomic fraction from blood in over 700 subjects. Hum Mol Genet. 2014;23:1175-85.

13. Florath I, Butterbach $\mathrm{K}$, Muller $\mathrm{H}$, Bewerunge-Hudler $\mathrm{M}$, Brenner $\mathrm{H}$. Crosssectional and longitudinal changes in DNA methylation with age: an epigenome-wide analysis revealing over 60 novel age-associated CpG sites. Hum Mol Genet. 2014:23:1186-201.

14. Reynolds LM, Taylor JR, Ding J, Lohman K, Johnson C, Siscovick D, et al. Age-related variations in the methylome associated with gene expression in human monocytes and T cells. Nat Commun. 2014;5:5366.

15. Xu Z, Taylor JA. Genome-wide age-related DNA methylation changes in blood and other tissues relate to histone modification, expression and cancer. Carcinogenesis. 2014;35:356-64.

16. Fernandez AF, Bayon GF, Urdinguio RG, Torano EG, Garcia MG, Carella A, et al. H3K4me1 marks DNA regions hypomethylated during aging in human stem and differentiated cells. Genome Res. 2015:25:27-40.

17. Issa JP. Aging and epigenetic drift: a vicious cycle. J Clin Invest. 2014;124: 24-9.

18. Marioni RE, Shah S, McRae AF, Chen BH, Colicino E, Harris SE, et al. DNA methylation age of blood predicts all-cause mortality in later life. Genome Biol. 2015;16:25.

19. Rimmelé P, Bigarella Carolina L, Liang R, Izac B, Dieguez-Gonzalez R, et al. Aging-like phenotype and defective lineage specification in SIRT1-deleted hematopoietic stem and progenitor cells. Stem Cell Reports. 2014;3:44-59.

20. Bauer M, Linsel G, Fink B, Offenberg K, Hahn AM, Sack U, et al. A varying T cell subtype explains apparent tobacco smoking induced single CpG hypomethylation in whole blood. Clin Epigenetics. 2015;7:81.

21. Zhang R, Chen H-Z, Liu D-P. The four layers of aging. Cell Systems. 2015;1: 180-6.

22. Okitsu CY, Hsieh CL. Sensitivity and specificity of immunoprecipitation of DNA containing 5-Methylcytosine. BMC Res Notes. 2015;8:102.

23. Schork AJ, Thompson WK, Pham P, Torkamani A, Roddey JC, Sullivan PF, et al. All SNPs are not created equal: genome-wide association studies reveal a consistent pattern of enrichment among functionally annotated SNPs. PLoS Genet. 2013;9, e1003449.

24. Welter D, MacArthur J, Morales J, Burdett T, Hall P, Junkins $H$, et al. The NHGRI GWAS Catalog, a curated resource of SNP-trait associations. Nucleic Acids Res. 2014;42:D1001-6.

25. Hindorff LA, Sethupathy P, Junkins HA, Ramos EM, Mehta JP, Collins FS, et al. Potential etiologic and functional implications of genome-wide association loci for human diseases and traits. Proc Natl Acad Sci U S A. 2009:106:9362-7.

26. Schaub MA, Boyle AP, Kundaje A, Batzoglou S, Snyder M. Linking disease associations with regulatory information in the human genome. Genome Res. 2012;22:1748-59.

27. Richards EJ. Inherited epigenetic variation-revisiting soft inheritance. Nat Rev Genet. 2006;7:395-401.

28. Bernstein BE, Birney E, Dunham I, Green ED, Gunter C, Snyder M. An integrated encyclopedia of DNA elements in the human genome. Nature. 2012;489:57-74

29. Domcke S, Bardet AF, Adrian Ginno P, Hartl D, Burger L, Schubeler D. Competition between DNA methylation and transcription factors determines binding of NRF1. Nature. 2015;528:575-9.

30. Ernst J, Kheradpour P, Mikkelsen TS, Shoresh N, Ward LD, Epstein CB, et al. Mapping and analysis of chromatin state dynamics in nine human cell types. Nature. 2011:473:43-9.

31. Hoffman MM, Ernst J, Wilder SP, Kundaje A, Harris RS, Libbrecht M, et al. Integrative annotation of chromatin elements from ENCODE data. Nucleic Acids Res. 2013:41:827-41.

32. Wang Y, Li X, Hu H. H3K4me2 reliably defines transcription factor binding regions in different cells. Genomics. 2014;103:222-8.

33. Irizarry RA, Ladd-Acosta C, Wen B, Wu Z, Montano C, Onyango P, et al. The human colon cancer methylome shows similar hypo- and hypermethylation at conserved tissue-specific CpG island shores. Nat Genet. 2009:41:178-86.

34. Doi A, Park $I H$, Wen B, Murakami P, Aryee MJ, Irizarry R, et al. Differential methylation of tissue- and cancer-specific CpG island shores distinguishes human induced pluripotent stem cells, embryonic stem cells and fibroblasts. Nat Genet. 2009;41:1350-3.

35. Wilson GA, Butcher LM, Foster HR, Feber A, Roos C, Walter L, et al. Humanspecific epigenetic variation in the immunological Leukotriene B4 Receptor (LTB4R/BLT1) implicated in common inflammatory diseases. Genome Med. $2014 ; 6: 19$

36. Andersson R, Gebhard C, Miguel-Escalada I, Hoof I, Bornholdt J, Boyd M et al. An atlas of active enhancers across human cell types and tissues. Nature. 2014:507:455-61.

37. Thomas-Chollier M, Hufton A, Heinig M, O'Keeffe S, Masri NE, Roider HG, et al. Transcription factor binding predictions using TRAP for the analysis of ChIP-seq data and regulatory SNPs. Nat Protoc. 2011;6:1860-9.

38. Machanick P, Bailey TL. MEME-ChIP: motif analysis of large DNA datasets. Bioinformatics. 2011;27:1696-7.

39. Tacutu R, Craig T, Budovsky A, Wuttke D, Lehmann G, Taranukha D, et al. Human Ageing Genomic Resources: integrated databases and tools for the biology and genetics of ageing. Nucleic Acids Res. 2013;41:D1027-33.

40. Lienert F, Wirbelauer C, Som I, Dean A, Mohn F, Schubeler D. Identification of genetic elements that autonomously determine DNA methylation states. Nat Genet. 2011:43:1091-7.

41. Small KS, Hedman AK, Grundberg E, Nica AC, Thorleifsson G, Kong A, et al. Identification of an imprinted master trans regulator at the KLF14 locus related to multiple metabolic phenotypes. Nat Genet. 2011;43:561-4.

42. Yuan $T$, Jiao Y, de Jong S, Ophoff RA, Beck S, Teschendorff AE. An integrative multi-scale analysis of the dynamic DNA methylation landscape in aging. PLoS Genet. 2015:11, e1004996.

43. Houseman EA, Accomando WP, Koestler DC, Christensen BC, Marsit CJ, Nelson $\mathrm{HH}$, et al. DNA methylation arrays as surrogate measures of cell mixture distribution. BMC Bioinformatics. 2012;13:86.

44. Roederer $M$, Quaye L, Mangino $M$, Beddall MH, Mahnke $Y$, Chattopadhyay $P$, et al. The genetic architecture of the human immune system: a bioresource for autoimmunity and disease pathogenesis. Cell. 2015;161:387-403.

45. Mohan S, Farley JR, Baylink DJ. Age-related changes in IGFBP-4 and IGFBP-5 levels in human serum and bone: implications for bone loss with aging. Prog Growth Factor Res. 1995;6:465-73.

46. Wei Z, Peddibhotla S, Lin H, Fang X, Li M, Rosen JM, et al. Early-onset aging and defective DNA damage response in Cdc14b-deficient mice. Mol Cell Biol. 2011;31:1470-7

47. Gjoneska E, Pfenning AR, Mathys H, Quon G, Kundaje A, Tsai LH, et al. Conserved epigenomic signals in mice and humans reveal immune basis of Alzheimer's disease. Nature. 2015:518:365-9.

48. Schultz MD, He Y, Whitaker JW, Hariharan M, Mukamel EA, Leung D, et al. Human body epigenome maps reveal noncanonical DNA methylation variation. Nature. 2015;523:212-6. 
49. Perez-Sieira S, Lopez M, Nogueiras R, Tovar S. Regulation of NR4A by nutritional status, gender, postnatal development and hormonal deficiency. Sci Rep. 2014;4:4264

50. Lin L, Nuotio-Antar AM, Ma X, Liu F, Fiorotto ML, Sun Y. Ghrelin receptor regulates appetite and satiety during aging in mice by regulating meal frequency and portion size but not total food intake. J Nutr. 2014:144:1349-55.

51. Gu M, Shen L, Bai L, Gao J, Marshall C, Wu T, et al. Heterozygous knockout of the Bmi-1 gene causes an early onset of phenotypes associated with brain aging. Age (Dordr). 2014;36:129-39.

52. Elks CE, Perry JR, Sulem P, Chasman DI, Franceschini N, He C, et al. Thirty new loci for age at menarche identified by a meta-analysis of genome-wide association studies. Nat Genet. 2010;42:1077-85.

53. Collado M, Blasco MA, Serrano M. Cellular senescence in cancer and aging Cell. 2007;130:223-33.

54. Barrio S, Gallardo M, Albizua E, Jimenez A, Rapado I, Ayala R, et al. Epigenomic profiling in polycythaemia vera and essential thrombocythaemia shows low levels of aberrant DNA methylation. J Clin Pathol. 2011;64:1010-3.

55. Thurman RE, Rynes E, Humbert R, Vierstra J, Maurano MT, Haugen E, et al. The accessible chromatin landscape of the human genome. Nature. 2012; 489:75-82.

56. Kim EK, Choi E-J. Pathological roles of MAPK signaling pathways in human diseases. Biochim Biophys Acta (BBA) - Mol Basis Dis. 2010;1802:396-405.

57. Stoffel W, Holz B, Jenke B, Binczek E, Gunter RH, Kiss C, et al. Delta6desaturase (FADS2) deficiency unveils the role of omega3- and omega6polyunsaturated fatty acids. EMBO J. 2008;27:2281-92.

58. Gu Y, Nakamura T, Alder H, Prasad R, Canaani O, Cimino G, et al. The t(4;11) chromosome translocation of human acute leukemias fuses the ALL-1 gene, related to Drosophila trithorax, to the AF-4 gene. Cell. 1992;71:701-8.

59. Zucker SN, Fink EE, Bagati A, Mannava S, Bianchi-Smiraglia A, Bogner PN, et al. Nrf2 amplifies oxidative stress via induction of KIf9. Mol Cell. 2014;53: 916-28.

60. Maurano MT, Humbert R, Rynes E, Thurman RE, Haugen E, Wang H, et al. Systematic localization of common disease-associated variation in regulatory DNA. Science. 2012;337:1190-5

61. Zhang W, Li J, Suzuki K, Qu J, Wang P, Zhou J, et al. Aging stem cells. A Werner syndrome stem cell model unveils heterochromatin alterations as a driver of human aging. Science. 2015;348:1160-3.

62. Stadler MB, Murr R, Burger L, Ivanek R, Lienert F, Scholer A, et al. DNAbinding factors shape the mouse methylome at distal regulatory regions. Nature. 2011:480:490-5.

63. Schubeler D. Function and information content of DNA methylation. Nature. 2015;517:321-6.

64. Wijetunga NA, Delahaye F, Zhao YM, Golden A, Mar JC, Einstein FH, et al. The meta-epigenomic structure of purified human stem cell populations is defined at cis-regulatory sequences. Nat Commun. 2014;5:5195.

65. Yang J, Yu L, Gaiteri C, Srivastava GP, Chibnik LB, Leurgans SE, et al. Association of DNA methylation in the brain with age in older persons is confounded by common neuropathologies. Int J Biochem Cell Biol. 2015;67: 58-64.

66. Ziller MJ, Gu H, Muller F, Donaghey J, Tsai LT, Kohlbacher O, et al. Charting a dynamic DNA methylation landscape of the human genome. Nature. 2013:500:477-81.

67. Liu Y, Aryee MJ, Padyukov L, Fallin MD, Hesselberg E, Runarsson A, et al. Epigenome-wide association data implicate DNA methylation as an intermediary of genetic risk in rheumatoid arthritis. Nat Biotech. 2013;31: 142-7.

68. Wagner W, Weidner Cl, Lin Q. Do age-associated DNA methylation changes increase the risk of malignant transformation? Bioessays. 2015:37:20-4.

69. Jost $\mathrm{E}$, Lin $\mathrm{Q}$, Weidner Cl, Wilop S, Hoffmann M, Walenda T, et al. Epimutations mimic genomic mutations of DNMT3A in acute myeloid leukemia. Leukemia. 2014;28:1227-34

70. Feinberg AP. Phenotypic plasticity and the epigenetics of human disease. Nature. 2007;447:433-40

71. Vockley CM, Guo C, Majoros WH, Nodzenski M, Scholtens DM, Hayes MG, et al. Massively parallel quantification of the regulatory effects of noncoding genetic variation in a human cohort. Genome Res. 2015;25:1206-14.

72. Bhatia G, Gusev A, Loh P-R, Vilhjálmsson BJ, Ripke S, Purcell S, et al. Haplotypes of common SNPs can explain missing heritability of complex diseases. bioRxiv. 2015. doi: 10.1101/022418
73. Bell CG, Finer S, Lindgren CM, Wilson GA, Rakyan VK, Teschendorff AE, et al. Integrated genetic and epigenetic analysis identifies haplotype-specific methylation in the FTO type 2 diabetes and obesity susceptibility locus. PLoS One. 2010;5, e14040.

74. Claussnitzer M, Dankel SN, Kim K-H, Quon G, Meuleman W, Haugen C, et al. FTO obesity variant circuitry and adipocyte browning in humans. N Engl J Med. 2015;373:895-907.

75. Moayyeri A, Hammond CJ, Valdes AM, Spector TD. Cohort Profile: TwinsUK and healthy ageing twin study. Int J Epidemiol. 2013;42:76-85.

76. Mamrut S, Avidan N, Staun-Ram E, Ginzburg E, Truffault F, Berrih-Aknin S, et al. Integrative analysis of methylome and transcriptome in human blood identifies extensive sex- and immune cell-specific differentially methylated regions. Epigenetics. 2015;10:943-57.

77. Li H, Durbin R. Fast and accurate short read alignment with BurrowsWheeler transform. Bioinformatics. 2009:25:1754-60.

78. Li H, Handsaker B, Wysoker A, Fennell T, Ruan J, Homer N, et al. The Sequence Alignment/Map format and SAMtools. Bioinformatics. 2009;25: 2078-9.

79. Chavez L, Jozefczuk J, Grimm C, Dietrich J, Timmermann B, Lehrach H, et al. Computational analysis of genome-wide DNA methylation during the differentiation of human embryonic stem cells along the endodermal lineage. Genome Res. 2010;20:1441-50.

80. Pruim RJ, Welch RP, Sanna S, Teslovich TM, Chines PS, Gliedt TP, et al. LocusZoom: regional visualization of genome-wide association scan results. Bioinformatics. 2010;26:2336-7.

81. Ihaka R, Gentleman R. R: A Language for Data Analysis and Graphics. J Comput Graph Stat. 1996;5:299-314.

82. Bates D, Maechler M, Bolker B, Walker S, Christensen RHB, Singmann H, Dai B, Eigen C, Rcpp L. Package 'Ime4'. 2015

83. Lemire M, Zaidi SHE, Ban M, Ge B, Aïssi D, Germain M, et al. Long-range epigenetic regulation is conferred by genetic variation located at thousands of independent loci. Nat Commun. 2015;6:6326.

84. Fujita PA, Rhead B, Zweig AS, Hinrichs AS, Karolchik D, Cline MS, et al. The UCSC Genome Browser database: update 2011. Nucleic Acids Res. 2011;39: D876-82.

85. Halachev K, Bast H, Albrecht F, Lengauer T, Bock C. EpiExplorer: live exploration and global analysis of large epigenomic datasets. Genome Biol. 2012;13:R96.

86. Gerstein MB, Kundaje A, Hariharan M, Landt SG, Yan KK, Cheng C, et al. Architecture of the human regulatory network derived from ENCODE data. Nature. 2012:489:91-100.

87. Siepel A, Bejerano G, Pedersen JS, Hinrichs AS, Hou M, Rosenbloom K, et al. Evolutionarily conserved elements in vertebrate, insect, worm, and yeast genomes. Genome Res. 2005:15:1034-50.

88. McLean CY, Bristor D, Hiller M, Clarke SL, Schaar BT, Lowe CB, et al. GREAT improves functional interpretation of cis-regulatory regions. Nat Biotechnol. 2010;28:495-501.

89. Gupta S, Stamatoyannopoulos JA, Bailey TL, Noble WS. Quantifying similarity between motifs. Genome Biol. 2007;8:R24.

90. Teschendorff AE, Marabita F, Lechner M, Bartlett T, Tegner J, Gomez-Cabrero D. A beta-mixture quantile normalization method for correcting probe design bias in Illumina Infinium 450 k DNA methylation data. Bioinformatics. 2013:29:189-96.

\section{Submit your next manuscript to BioMed Central and we will help you at every step:}

- We accept pre-submission inquiries

- Our selector tool helps you to find the most relevant journal

- We provide round the clock customer support

- Convenient online submission

- Thorough peer review

- Inclusion in PubMed and all major indexing services

- Maximum visibility for your research

Submit your manuscript at www.biomedcentral.com/submit 